\title{
The trait anger affects conflict inhibition: a Go/Nogo ERP study
}

\author{
Yong Liu, Xianghong Zhan *, Wei Li, Heyun Han, Huixia Wang, Junlin Hou, Guoli Yan and \\ Youjie Wang
}

Basic Disciplines of Integrated Traditional Chinese and Western Medicine, School of Basic Medicine, Henan University of Traditional Chinese Medicine, Zhengzhou, China

Edited by:

Francesco Di Russo, University of

Rome "Foro Italico," Italy

\section{Reviewed by:}

Giuliana Lucci, IRCCS Santa Lucia of Rome, Italy

Annalisa Bello, University of Rome

"Sapienza," Italy

*Correspondence:

Xianghong Zhan, Basic Disciplines of Integrated Traditional Chinese and

Western Medicine, School of Basic

Medicine, Henan University of

Traditional Chinese Medicine,

Jinshui East Road 1, Zhengzhou,

China

e-mail: zhan20140923@163.com;

zxh371@163.com
To explore the time course of inhibitory control in high trait anger individuals, we recorded and analyzed ERP data relevant to visual Go/Nogo task in high and low trait anger participants. Compared with low trait anger participants, high trait anger participants revealed faster RTs in the Go/Nogo task. The nogo effect of N2 related to conflict monitoring was similar between two groups. While the P3go was larger in high than low trait anger groups, the P3nogo did not differ between two groups. This induced the smaller nogo effect of P3 in high than that in low trait anger group, which is closely related to the actual inhibition of the motor system. These data suggest the reduced later stage of inhibitory processes in high trait anger individuals, implicating the dysfunction of inhibitory control.

Keywords: trait anger, conflict inhibition, Go/Nogo, N2, P3

\section{INTRODUCTION}

As a common mood in everyday life, anger is considered to be the core mechanism of mood disorders, anxiety and aggression and closely associated with impulsive aggression, damage behavior, etc (Wilkowski and Robinson, 2010). Long-term anger can bring serious impact to the body health and social relations (Deffenbacher et al., 2003; Bettencourt et al., 2006; Shorey et al., 2011). Generally, trait anger is defined "in terms of individual differences in the disposition to perceive a wide range of situations as annoying or frustrating and by the tendency to respond to such situations with elevations in state anger" (Forgays et al., 1997). High levels of trait anger predict quite a few health outcomes (Robinson et al., 2012). For example, angry individuals suffer from excessive cardiovascular reactivity (Suarez et al., 1998), higher blood pressure (Suls et al., 1995), many other physical problems (Williams et al., 2002), and psychological health problems, such as borderline personality (Distel et al., 2012), attempted suicide (Daniel et al., 2009). Especially, angry individuals damage their social relationships (Baron et al., 2007), receive less social support (Smith et al., 2004), and have high divorce rate (Roberts et al., 2007).

Several theoretical perspectives underlying the characteristic trait of angry individuals have been proposed (Wilkowski and Robinson, 2010; Owen, 2011). Recent studies revealed that individuals with superior inhibitory control abilities may be able to override their automatic tendencies toward anger and aggression. For example, Gagne et al assessed the associations between anger and inhibitory control in a twin sample from 12 to 36 months of age and found that twins with low levels of inhibitory control had high levels of anger (Gagne and Goldsmith, 2011). As a core component of inhibitory control, response inhibition is generally defined as the ability to adaptively suppress behavior when environmental contingences demand this (Luijten et al., 2011). Recently, Wilkowski et al. suggested that anger and reactive aggression may represent a more specific aspect of personality and psychopathology directly relevant to response inhibition. Thus, individuals may mobilize the cognitive resources needed for response inhibition when it serves their goals, and they may allow these same resources to lay dormant when they have little motivation to contain inappropriate responses. These findings suggested that low trait anger individuals mobilize these resources when they encounter angry expressions; while high trait anger individuals allow these resources to lie dormant under the same circumstances (Wilkowski, 2012). Recent neuroimaging research found that trait anger related to increased left frontal cortical activation and that this relationship was not due to anger being regarded as a positive feeling (Pessoa, 2010). In addition, there was evidence that trait anger was inversely associated with the strength of resting-state functional connectivity (RSFC) between the amygdala and the contralateral middle orbitofrontal cortex, especially for the right amygdale- left orbitofrontal connectivity (Fulwiler et al., 2012). To this end, previous findings showed that the difference of the inhibitory control might be the foundation of trait anger; however, the time course of this inhibitory control had never been reported for trait anger, which will be investigated by recording and analyzing ERPs in response to Go/Nogo task relevant to inhibitory control. 
The Go/Nogo paradigm is widely used for the assessment of inhibitory control (Pfefferbaum et al., 1985). Two ERP components with a frontal-central distribution, N2nogo and P3nogo, are enhanced in response to Nogo trails over Go trials, reflecting changes in brain activity related to response inhibition (Yang et al., 2009). The N2nogo is a negative wave that emerges approximately 200-300 ms after stimulus presentation and the P3nogo is a positive wave that emerges $300-600 \mathrm{~ms}$ after stimulus onset. Geczy et al. suggested that increased N2 amplitude in response to Nogo stimuli after Go cues might be related to increased efforts to activate the response inhibition system and to interrupt preparations for response execution (Géczy et al., 1999). Converging evidence suggested that the $\mathrm{N} 2$ nogo amplitude is a valuable measure for response inhibition. In contrast, Nieuwenhuis suggested that the N2nogo reflects response conflict rather than inhibition because it was enhanced for low-frequency stimuli and was localized to the anterior cingulated cortex (Nieuwenhuis et al., 2003). Although the overlapping of movement-related activities may influence the difference between Go and Nogo ERPs within this time range, the $\mathrm{P} 3$ nogo is generally related to the later stage of the inhibition process that is closely related to the actual inhibition of the motor system in the premotor cortex (Bokura et al., 2001; Kok et al., 2004; Smith et al., 2008; Verleger et al., 2009). In addition, it has been suggested that the difference waves (Nogo- minus GoERPs) would reflect the Go/Nogo effect and further specifically reflect frontal inhibitory functioning, that is the difference waves of N2 (N2d) and P3 (P3d), which were defined as the results of N2go subtracted from the N2nogo and P3go subtracted from the P3nogo, respectively (Bokura et al., 2001). Although there are still many controversial reports, it was widely accepted that these components elicited by Nogo stimuli, i.e., N2nogo (N2d) and P3nogo (P3d), are associated with two aspects of inhibitory control, i.e., conflict monitoring and response inhibition (Yang et al., 2009). To date, few studies investigated response inhibition in high and low trait anger by use of ERPs, which will be conducted in the present visual Go/Nogo paradigm.

\section{METHODS \\ PARTICIPANTS}

Two thousand six hundred and forty two participants (1533 female; $25.3 \pm 4.8$ years) completed the STAXI-2 (State-Trait Anger Expression Inventory 2) ${ }^{1}$ and the handedness scale (Spielberger, 1988). Trait anger was assessed by the trait anger scale (TAS) ${ }^{2}$ and the TAS score for all participants was $17.81 \pm$ 3.75. According the TAS scores of each participant, we selected

\footnotetext{
${ }^{1}$ STAXI - State-Trait Anger Expression Inventory is a 44-item questionnaire and is extensively utilized in research on anger. It distinguishes between the three modes of anger expression: anger-out, anger-in, and anger-control. Anger-out refers to a tendency to express anger through either verbal or physical behaviors. Anger-in or suppressed anger refers to the tendency to hold one's anger on the inside without any outlet. Anger-control refers to the tendency to engage in behaviors intended to reduce overt anger expression (Spielberger, 1988).

${ }^{2}$ Generally, State-Trait Anger Scale (STAS) includes 10 items and is built upon two subscales: State anger, defined as an emotional state or condition that consists of subjective feelings of tension, annoyance, irritation, fury and rage; Trait anger defined in terms of individual differences in the frequency that
}

those subjects with higher TAS score for the high anger trait group (TAS score $\geq 22$ ) and lower TAS score for the low anger trait group (TAS score $\leq 14$ ). To address the present question, then, we selected randomly 16 participants (eight female; 24.3 \pm 2.8 years) from the high anger trait group and 16 participants from the low anger trait group (eight female; $24.1 \pm 3.4$ years), respectively. All of 32 participants were right handed and free of medication for at least $24 \mathrm{~h}$ before testing, with normal or corrected-to-normal vision, without history of head trauma or other medical conditions that could cause cognitive impairment (Yang et al., 2009). The study was conducted in accordance with the Declaration of Helsinki and all procedures were carried out with the adequate understanding and written informed consent of the subjects. The study protocol was approved by the Ethics Committee of the Institute of Psychology of the HACTCM.

\section{STIMULI AND PROCEDURE}

Visual stimuli included single and double triangles in gray background, presented in the center of a computer screen (light degree $=60 \mathrm{~cd} / \mathrm{m}^{2}$ ). Participants were seated in a semi-dark room, facing a monitor place $75 \mathrm{~cm}$ from their eyes, with a visual angle of $4^{\circ} \times 4^{\circ}$. There were four blocks with 60 Go (double triangles) and 40 Nogo (single triangle) stimuli for each. The participants were instructed to respond by pressing a button as quickly as possible after the Go stimuli appeared and to withhold the response when the Nogo stimuli appeared. Each stimulus was presented for $100 \mathrm{~ms}$, with the mean interstimulus intervals (ISI) being $1200 \mathrm{~ms}$ (randomly between 1000 and $1400 \mathrm{~ms}$ ). The hand to press button was counterbalanced across the participants. Before EEG recording, participants performed one practice block consisting of 40 Go and Nogo trials. During the experiment, participants were instructed to watch the center of the screen, relax, and minimize eye blinks or body movements.

\section{EEG RECORDING AND ANALYSIS}

Based on the present aim, EEG signals were continuously recorded (band pass $0.05-100 \mathrm{~Hz}$, sampling rate $500 \mathrm{~Hz}$ ) using 32-channel Ag/AgCl electrodes cap (10-20 International System; Quick Cap, www.neuroscan.com) by NuAmps amplifier, referenced to the left mastoid (right mastoid as recording site). VEOG and HEOG were recorded with two pairs of electrodes, one placed above and below right eye, and the other $10 \mathrm{~mm}$ from the lateral canthi. Electrode impedance was maintained below $5 \mathrm{k} \Omega$ throughout the experiment.

We used EMSE 5.5 software (www.sourcesignal.com) to analyze the data off-line. EEG data were re-referenced to the bimastoid average reference. EOG artifacts were corrected offline. The EEG was segmented into the epoch from $200 \mathrm{~ms}$ pre-stimulus to $1000 \mathrm{~ms}$ post-stimulus. Trials contaminated by amplifier clipping, bursts of electromyographic activity, or peak-to-peak deflection exceeding $\pm 100 \mu \mathrm{v}$ were excluded from averaging. The EEG segments were averaged separately for target and standard

State anger was experienced over time. We recorded the trait anger scale in the present study. (Spielberger et al., 1983) 
stimuli. The number of average trials left after removal of the artifacts was 130 (Nogo) and 202 (Go) for low and 125 (Nogo) and 208 (Go) for high trait anger, respectively.

Behavioral results (RT and accuracy) were compared by $t$-test to explore the group difference between high and low trait anger groups. For ERPs data, according to the frontal-central scalp distribution of $\mathrm{N} 2$ nogo and $\mathrm{P} 3$ nogo components, we focused on the analysis at frontal-central electrode sites $(\mathrm{Fz}, \mathrm{FCz}, \mathrm{Cz})$. The $\mathrm{N} 2$ component was quantified as the most negative amplitude within a 200 to $300 \mathrm{~ms}$ window following stimulus onset. The P3 component was quantified as the most positive amplitude within 300 to $600 \mathrm{~ms}$ following the $\mathrm{N} 2$ peak. In order to highlight the Nogo effect, difference waves (N2d and P3d; Nogo- minus GoERPs) were computed (Yang et al., 2009). The measurements of peak latencies and amplitudes of N2 and P3 components were subjected to Three-Way repeated measures ANOVA with Stimulus (Go, Nogo) and Site (Fz, FCz, Cz) as within-subject factors and Group (high, low) as between-subject factor. A Site (Fz, $\mathrm{FCz}, \mathrm{Cz}) \times$ Group (high, low) repeated measures ANOVA was performed for the measurements of N2d and P3d components. The Geisser-Greenhouse correction was used for any repeated measures containing more than one degree of freedom in the numerator (Geisser and Greenhouse, 1958).

\section{RESULTS}

\section{BEHAVIORAL DATA}

Behavioral data indicated that RTs was faster in the high score group $(302 \mathrm{~ms})$ than that in the low score group $(328 \mathrm{~ms}$; $t=2.54, p=0.017)$. The accuracy did not differ between two groups (96.3 and $95.6 \%$ for high and low score groups, respectively; $t=0.66, p=0.51)$.

\section{ERPs DATA}

Figure 1 illustrates the grand averages for the Go and Nogo stimuli in the high and low trait anger groups, respectively. Compared with the ERP waveforms elicited by Go stimuli, Nogo stimuli elicited larger frontal-central N2 (i.e., N2nogo) and P3 (i.e., P3nogo) components, regardless of high or low score group.

Because across Go and Nogo stimuli we did not find any significant group differences for latencies of $\mathrm{N} 2\left[F_{(1,26)}=0.69\right.$, $p=0.41]$ and P3 $\left[F_{(1,26)}=0.15, p=0.69\right]$ components, the following analysis focused on the amplitude of each component.

The N2 was slightly larger for low $(-4.5 \mu \mathrm{V})$ than high $\left[-2.7 \mu \mathrm{V} ; F_{(1,26)}=0.99, p=0.33\right]$ trait anger groups. Across participants' groups, the N2 amplitudes were overall larger for Nogo $(-5.2 \mu \mathrm{V})$ than for Go stimuli $\left[-2.0 \mu \mathrm{V} ; F_{(1,26)}=12.89\right.$, $p=0.005]$. The main effect for Site was also significant, $F_{(2,52)}=10.81, p<0.001$, with the largest N2 $(-4.1 \mu \mathrm{V})$ at FCz site. No interactions were significant $(p s>0.1)$.

Similar to the analysis of $\mathrm{N} 2$ component, the P3 amplitudes were overall larger for Nogo $(-5.2 \mu \mathrm{V})$ than for Go stimuli $\left[-2.0 \mu \mathrm{V} ; F_{(1,26)}=12.90, p=0.004\right]$. The main effect for Site was also significant, $F_{(2,52)}=9.88, p<0.001$, with the largest P3 $(10.4 \mu \mathrm{V})$ at $\mathrm{Cz}$ site. Importantly, a Group $\times$ Stimulus interaction was found, $F_{(1,26)}=4.34, p=0.041$. Post-hoc analysis indicated that the P3 elicited by Go stimuli was significantly reduced in low $(7.2 \mu \mathrm{V})$ over high score group $[9.9 \mu \mathrm{V}$;

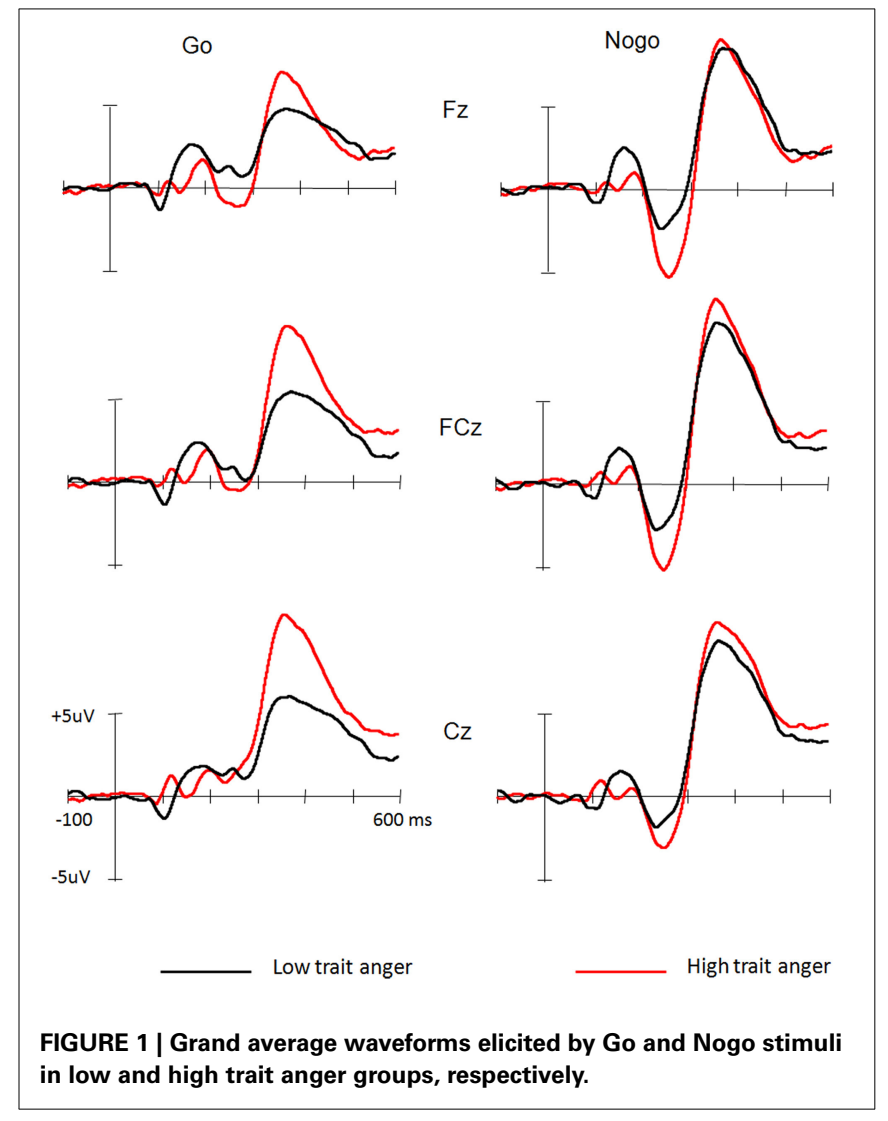

$\left.F_{(1,26)}=5.06, p=0.033\right]$, whereas the P3nogo did not differ between two groups [10.4 and $11.4 \mu \mathrm{V}$ for low and high score groups, respectively; $F_{(1,26)}=0.41, p=0.53$ ], and that, while the nogo effect of P3 component was significant in low score group $\left[F_{(1,26)}=16.10, p<0.001\right]$, it was not evident in high score group $\left[F_{(1,26)}=1.14, p=0.29\right]$.

For the analysis of difference waveforms related to inhibition effects (Figure 2), the ANOVA revealed that the amplitude of N2d was similar between two groups $[-2.5$ and $-3.3 \mu \mathrm{V}$ for high and low trait anger groups, respectively; $\left.F_{(1,26)}=2.42, p=0.132\right]$, whereas the P3d was significantly reduced in high $(0.4 \mu \mathrm{V})$ than low $(2.2 \mu \mathrm{V})$ trait anger group $\left[F_{(1,26)}=6.83, p=0.024\right]$.

\section{DISCUSSION}

The main aim of the present study was to investigate the effects of the trait anger on response inhibition. Compared with low trait anger participants, high trait anger participants revealed faster RTs in the Go/Nogo task. Across groups, the nogo stimuli elicited larger N2 and P3 components than did the go stimuli. Although the nogo effect of N2 was similar between low and high trait anger groups, the P3 elicited by Go stimuli was larger in high than low trait anger groups and the P3nogo did not differ between two groups. This induced smaller P3d component in high over low trait anger groups.

Consistent with the present RT data, Parrott et al reported that high levels of trait anger displayed facilitative biases in the processing of semantic anger-related stimuli (Parrott et al., 2005). However, this effect was not found widely (Wenzel and Lystad, 

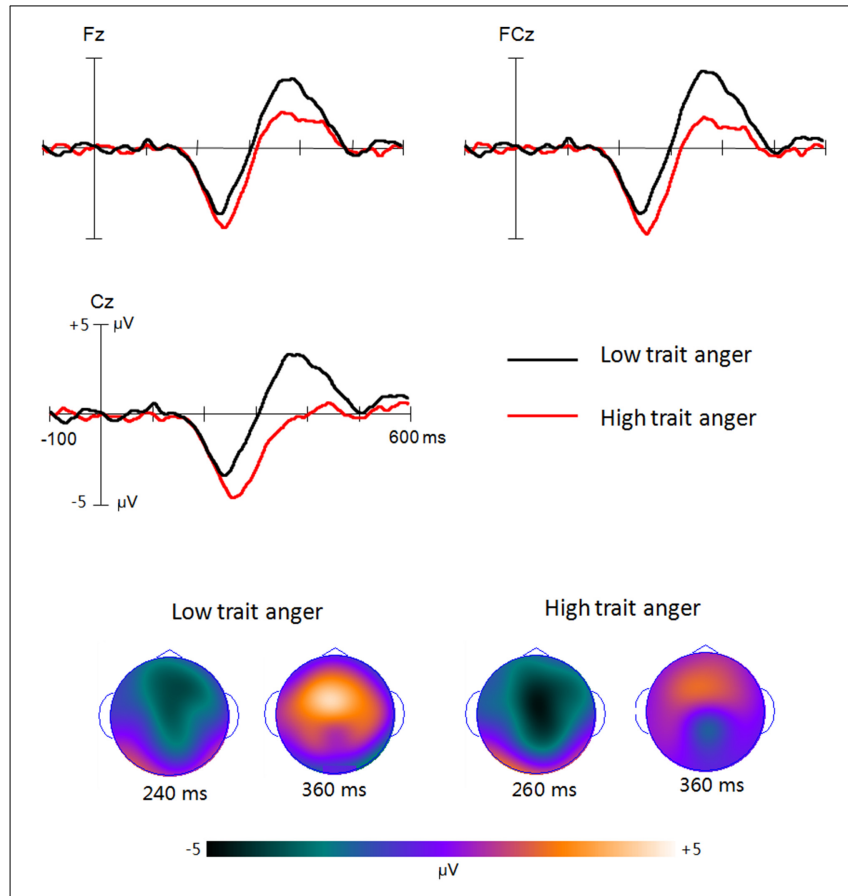

FIGURE 2 | The difference waveforms between Nogo and Go ERPs in low and high trait anger groups, respectively, as well as the 2D scalp distribution topography of the peak amplitudes of N2 and P3 components.

2005). Indeed, in previous studies the stimuli with anger information were presented to participants and the state anger after the experiment was not measured; hence, it is hard to contribute the change of state anger and/or trait anger to the RT difference between participants with low and high levels of trait anger (Wenzel and Lystad, 2005). The present experiment presented a simple triangle shape without any emotional information, which would not change the state anger of participants. The fast performance in participants with high level of trait anger is indeed consistent with the idea that anger is associated with greater impulsiveness (Ramírez and Andreu, 2006; Jaworska et al., 2012).

Consistent with previous studies, greater N2 amplitudes were observed in Nogo than in Go trials, regardless of the trait anger. There was evidence that the N2nogo amplitude is a valuable indicator for the measurement of response conflict. The present fact that the N2nogo as well as N2d did not differ between high and low angry persons indicated that the response conflict especially the detection of conflict information is not modulated by the trait anger.

Although there was evidence that the P3nogo component may not necessarily represent inhibition of a response (Falkenstein et al., 1999; Fallgatter and Strik, 1999), it is accepted that the P3nogo is related to the outcome of the inhibition process and reflects the conflict inhibition processing (Overtoom et al., 2002; Dimoska et al., 2003). The reduced P3d amplitudes in the present high trait anger group indicated the impaired response inhibition. Supporting this view, one recent report showed that the anger group had more false alarms overall, indicating impaired response inhibition (Jaworska et al., 2012). In addition, the increased right cortical activation during the initial portion of CPT existed in the anger group, perhaps reflecting greater engagement of frontal circuits (i.e., effort) during initial stages of the task compared to controls, revealing a hyper-vigilant state in anger group, which may interfere with effective attention control and decrease inhibition (Jaworska et al., 2012).

It should be noted here that the reduced $\mathrm{Pd} 3$ in high trait anger group is indeed due to the decreased P3go in low trait anger group. In line with recent reports (Shucard et al., 2008), the present findings implicated that the hyper-arousal reflected by higher amplitude of target P3 might be a common feature of high trait anger participants. Generally, the hyper-arousal plays the primary role in producing an enhanced P3 to Go stimuli, and the high score individuals cannot mobilize more inhibitory resources on Nogo stimuli. Our behavioral findings of faster response to Go stimuli in the high trait anger group supported this hypothesis as well. It is reasonable that, in the cognitive processes from stimuli to response, high trait anger individuals have an inferior inhibitory control and then make a quick emotional response.

In sum, the present study explored the time course of inhibitory control in high trait anger individuals by recording and analyzing ERP data relevant to visual Go/Nogo task. While, the nogo effect of N2 related to conflict monitoring was similar between two groups, the nogo effect of P3 closely related to the actual inhibition of the motor system was smaller in high than that in low trait anger group. These data suggest the reduced later stage of inhibitory processes in high trait anger individuals, implicating the dysfunction of inhibitory control.

\section{ACKNOWLEDGMENTS}

We would like to thank the financial support of National Natural Science Foundation of China (No. 30772686, No. 30973695, No. 81173148, and No. 81473556).

\section{REFERENCES}

Baron, K. G., Smith, T. W., Butner, J., Nealey-Moore, J., Hawkins, M. W., and Uchino, B. N. (2007). Hostility, anger, and marital adjustment: concurrent and prospective associations with psychosocial vulnerability. J. Behav. Med. 30, 1-10. doi: 10.1007/s10865-006-9086-Z

Bettencourt, B. A., Talley, A., Benjamin, A. J., and Valentine, J. (2006). Personality and aggressive behavior under provoking and neutral conditions: a metaanalytic review. Psychol. Bull. 132, 751-777. doi: 10.1037/0033-2909.132.5.751

Bokura, H., Yamaguchi, S., and Kobayashi, S. (2001). Electrophysiological correlates for response inhibition in a Go/NoGo task. Clin. Neurophysiol. 112, 2224-2232. doi: 10.1016/S1388-2457(01)00691-5

Daniel, S. S., Goldston, D. B., Erkanli, A., Franklin, J. C., and Mayfield, A. M. (2009). Trait anger, anger expression, and suicide attempts among adolescents and young adults: a prospective study. J. Clin. Child. Adolesc. Psychol. 38, 661-671. doi: 10.1080/15374410903103494

Deffenbacher, J. L., Deffenbacher, D. M., Lynch, R. S., and Richards, T. L. (2003). Anger, aggression, and risky behavior: a comparison of high and low anger drivers. Behav. Res. Ther. 41, 701-718. doi: 10.1016/S0005-7967(02)00046-3

Dimoska, A., Johnstone, S. J., Barry, R. J., and Clarke, A. R. (2003). Inhibitory motor control in children with attention-deficit/hyperactivity disorder: eventrelated potentials in the stop-signal paradigm. Biol. Psychiatry 54, 1345-1354. doi: 10.1016/S0006-3223(03)00703-0

Distel, M. A., Roeling, M. P., Tielbeek, J. J., van Toor, D., Derom, C. A., Trull, T. J., et al. (2012). The covariation of trait anger and borderline personality: a bivariate twin-siblings study. J. Abnorm. Psychol. 121, 458-466. doi: $10.1037 / \mathrm{a} 0026393$ 
Falkenstein, M., Hoormann, J., and Hohnsbein, J. (1999). ERP components in Go/Nogo tasks and their relation to inhibition. Acta Psychol. (Amst.) 101, 267-291. doi: 10.1016/S0001-6918(99)00008-6

Fallgatter, A. J., and Strik, W. K. (1999). The NoGo-anteriorization as a neurophysiological standard-index for cognitive response control. Int. J. Psychophysiol. 32, 233-238. doi: 10.1016/S0167-8760(99)00018-5

Forgays, D. G., Forgays, D. K., and Spielberger, C. D. (1997). Factor structure of the State-Trait Anger Expression Inventory. J. Pers. Assess. 69, 497-507. doi: 10.1207/s15327752jpa6903_5

Fulwiler, C. E., King, J. A., and Zhang, N. (2012). Amygdala-orbitofrontal restingstate functional connectivity is associated with trait anger. Neuroreport 23, 606-610. doi: 10.1097/WNR.0b013e3283551cfc

Gagne, J. R., and Goldsmith, H. H. (2011). A longitudinal analysis of anger and inhibitory control in twins from 12 to 36 months of age. Dev. Sci. 14, 112-124. doi: 10.1111/j.1467-7687.2010.00969.x

Géczy, I., Czigler, I., and Balázs, L. (1999). Effects of cue information on response production and inhibition measured by event-related potentials. Acta Physiol. Hung. 86, 37-44.

Geisser, S., and Greenhouse, S. W. (1958). An extension of Box's results on the use of the F distribution in multivariate analysis. Ann. Math. Stat. 29, 885-891. doi: 10.1214/aoms/1177706545

Jaworska, N., Berrigan, L., Fisher, D., Ahmed, A. G., Gray, J., Bradford, J., et al. (2012). A pilot study of electrocortical activity in dysfunctional anger: decreased frontocortical activation, impaired attention control, and diminished behavioral inhibition. Aggress. Behav. 38, 469-480. doi: 10.1002/ab.21449

Kok, A., Ramautar, J. R., de Ruiter, M. B., Band, G. P., and Ridderinkhof, K. R. (2004). ERP components associated with successful and unsuccessful stopping in a stop-signal task. Psychophysiology 41, 9-20. doi: 10.1046/j.14698986.2003.00127.x

Luijten, M., Littel, M., and Franken, I. H. (2011). Deficits in inhibitory control in smokers during a Go/NoGo task: an investigation using eventrelated brain potentials. PLOS ONE 6:e18898. doi: 10.1371/journal.pone. 0018898

Nieuwenhuis, S., Yeung, N., van den Wildenberg, W., and Ridderinkhof, K. R. (2003). Electrophysiological correlates of anterior cingulate function in a go/nogo task: effects of response conflict and trial type frequency. Cogn. Affect. Behav. Neurosci. 3, 17-26. doi: 10.3758/CABN.3.1.17

Overtoom, C. C. E., Kenemans, J. L., Verbaten, M. N., Kemner, C., van der Molen, M. W., van Engeland, H., et al. (2002). Inhibition in children with attentiondeficit/hyperactivity disorder: a psychophysiological study of the stop task. Biol. Psychiatry 51, 668-676. doi: 10.1016/S0006-3223(01)01290-2

Owen, J. M. (2011). Transdiagnostic cognitive processes in high trait anger. Clin. Psychol. Rev. 31, 193-202. doi: 10.1016/j.cpr.2010.10.003

Parrott, D. J., Zeichner, A., and Evces, M. (2005). Effect of trait anger on cognitive processing of emotional stimuli. J. Gen. Psychol. 132, 67-80. doi: 10.3200/GENP.132.1.67-80

Pessoa, L. (2010). Emergent processes in cognitive-emotional interactions. Dialogues Clin. Neurosci. 12, 433-448.

Pfefferbaum, A., Ford, J. M., Weller, B. J., and Kopell, B. S. (1985). ERPs to response production and inhibition. Electroencephalogr. Clin. Neurophysiol. 60, 423-434. doi: 10.1016/0013-4694(85)91017-X

Ramírez, J. M., and Andreu, J. M. (2006). Aggression, and some related psychological constructs (anger, hostility, and impulsivity); some comments from a research project. Neurosci. Biobehav. Rev. 30, 276-291. doi: 10.1016/j.neubiorev.2005.04.015

Roberts, B. W., Kuncel, N. R., Shiner, R., Caspi, A., and Goldberg, L. R. (2007). The power of personality: The comparative validity of personality traits, socioeconomic status, and cognitive ability for predicting important life outcomes. Perspect. Psychol. Sci. 2, 313-345. doi: 10.1111/j.1745-6916.2007. 00047.x

Robinson, M. D., Wilkowski, B. M., Meier, B. P., Moeller, S. K., and Fetterman, A. K. (2012). Counting to ten milliseconds: low-anger, but not high-anger, individuals pause following negative evaluations. Cogn. Emot. 26, 261-281. doi: $10.1080 / 02699931.2011 .579088$
Shorey, R. C., Brasfield, H., Febres, J., and Stuart, G. L. (2011). The association between impulsivity, trait anger, and the perpetration of intimate partner and general violence among women arrested for domestic violence. J. Interpers. Violence 26, 2681-2697. doi: 10.1177/0886260510388289

Shucard, J. L., McCabe, D. C., and Szymanski, H. (2008). An event-related potential study of attention deficits in posttraumatic stress disorder during auditory and visual Go/NoGo continuous performance tasks. Biol. Psychol. 79, 223-233. doi: 10.1016/j.biopsycho.2008.05.005

Smith, J. L., Johnstone, S. J., and Barry, R. J. (2008). Movement-related potentials in the Go/NoGo task: the $\mathrm{P} 3$ reflects both cognitive and motor inhibition. Clin. Neurophysiol. 119, 704-714. doi: 10.1016/j.clinph.2007.11.042

Smith, T. W., Glazer, K., Ruiz, J. M., and Gallo, L. C. (2004). Hostility, anger, aggressiveness, and coronary heart disease: an interpersonal perspective on personality, emotion, and health. J. Pers. 72, 1217-1270. doi: 10.1111/j.14676494.2004.00296.x

Spielberger, C. D. (1988). Manual for the State-Trait Anger Expression Inventory (STAXI). Odessa, FL: Psychological Assessment Resources.

Spielberger, C. D., Jacobs, G., Russel, S., and Crane, R. S. (1983). "Assessment of anger: the state-trait anger scale," in Advances in Personality Assessment, eds C. D. Spielberger and J. N. Butcher (Hillsdale, NJ: Lawrence Erlbaum Associates Inc.), 159-187.

Suarez, E. C., Kuhn, C. M., Schanberg, S. M., Williams, R. B. Jr., and Zimmermann, E. A. (1998). Neuroendocrine, cardiovascular, and emotional responses of hostile men: the role of interpersonal challenge. Psychosom. Med. 60, 78-88. doi: 10.1097/00006842-199801000-00017

Suls, J., Wan, C. K., and Costa, P. T. Jr. (1995). Relationship of trait anger to resting blood pressure: a meta-analysis. Health Psychol. 14, 444-456. doi: 10.1037/02786133.14.5.444

Verleger, R., Paehge, T., Kolev, V., Yordanova, J., and Jaœkowski, P. (2009). On the relation of movement-related potentials to the go/no-go effect on P3. Biol. Psychol. 73, 298-313. doi: 10.1016/j.biopsycho.2006.05.005

Wenzel, A., and Lystad, C. (2005). Interpretation biases in angry and anxious individuals. Behav. Res. Ther. 43, 1045-1054. doi: 10.1016/j.brat.2004. 02.009

Wilkowski, B. M. (2012). Responding to social signals for response inhibition: a psychological process underlying trait anger. Soc. Psychol. Pers. Sci. 3, 72-78. doi: 10.1177/1948550611409246

Wilkowski, B. M., and Robinson, M. D. (2010). The anatomy of anger: an integrative cognitive model of trait anger and reactive aggression. J. Pers. 78, 9-38. doi: 10.1111/j.1467-6494.2009.00607.x

Williams, J. E., Nieto, F. J., Sanford, C. P., Couper, D. J., and Tyroler, H. A. (2002). The association between trait anger and incident stroke risk: the Atherosclerosis Risk in Communities (ARIC) Study. Stroke 33, 13-19. doi: 10.1161/hs0102.101625

Yang, B., Yang, S., Zhao, L., Yin, L., Liu, X., and An, S. (2009). Event-related potentials in a Go/Nogo task of abnormal response inhibition in heroin addicts. Sci. China C Life Sci. 52, 780-788. doi: 10.1007/s11427-009-0106-4

Conflict of Interest Statement: The authors declare that the research was conducted in the absence of any commercial or financial relationships that could be construed as a potential conflict of interest.

Received: 23 September 2014; accepted: 30 December 2014; published online: 21 January 2015.

Citation: Liu Y, Zhan X, Li W, Han H, Wang H, Hou J, Yan G and Wang Y (2015) The trait anger affects conflict inhibition: a Go/Nogo ERP study. Front. Hum. Neurosci. 8:1076. doi: 10.3389/fnhum.2014.01076

This article was submitted to the journal Frontiers in Human Neuroscience.

Copyright (C) 2015 Liu, Zhan, Li, Han, Wang, Hou, Yan and Wang. This is an openaccess article distributed under the terms of the Creative Commons Attribution License (CC BY). The use, distribution or reproduction in other forums is permitted, provided the original author(s) or licensor are credited and that the original publication in this journal is cited, in accordance with accepted academic practice. No use, distribution or reproduction is permitted which does not comply with these terms. 